\title{
Outcome Evaluation of the You Can Do It Initiative to Promote Exclusive Breastfeeding Among Women Enrolled in the New York State WIC Program by Race/Ethnicity
}

Lynn S. Edmunds, DrPH, MS, RD; Furrina F. Lee, PhD; Johanna D. Eldridge, BS; Jackson P. Sekhobo, PhD, MPA

\begin{abstract}
Objective: To evaluate the effectiveness of You Can Do It at improving exclusive breastfeeding (BF) among New York State women enrolled in Special Supplemental Nutrition Program for Women, Infants, and Children (WIC).

Design: Quasi-experimental study, September, 2013 through February, 2016.

Setting: Multicomponent intervention paired with a yearlong learning community in 12 clinics.

Participants: Women who were enrolled in WIC during the first trimester, intended to breastfeed or were undecided, and continued in WIC after delivery, comprised 1 baseline cohort $(\mathrm{n}=688)$ and 2 intervention cohorts: Breastfeeding Attrition Prediction Tool (BAPT) (consented, $\mathrm{n}=362$ ) and non-BAPT (declined, $\mathrm{n}=408)$.

Intervention: The BAPT was offered to all eligible women in the intervention enrollment period. Consenting women received multiple counseling sessions tailored to individual BAPT results throughout pregnancy and were contacted promptly after delivery.

Main Outcome Measure(s): Prevalence of exclusive BF at 7, 30, and 60 days.

Analysis: Multivariate logistic regression, stratified by race/ethnicity. Statistical significance set at $P<.05$.

Results: Prevalence of exclusive BF at 7 and 30 days was significantly higher among BAPT women compared with non-BAPT or baseline cohorts. Non-Hispanic black and Hispanic women in the BAPT cohort achieved significantly higher exclusive BF rates at 30 and 60 days compared with those in non-BAPT and baseline cohorts. Conclusions and Implication: The initiative seems to be effective at increasing exclusive BF, particularly among non-Hispanic black and Hispanic women in the New York State WIC program.

Key Words: WIC program, BAPT, tailored breastfeeding counseling, exclusive breastfeeding, outcome evaluation (J Nutr Educ Behav. 2017;49:S162-S168.)
\end{abstract}

Accepted May 11, 2017.

\section{INTRODUCTION}

Breastfeeding (BF) is widely accepted as the optimal method of infant feeding to convey short-term and long-term health benefits to both the mother and the child. ${ }^{1,2}$ Accordingly, BF promotion is a principal objective of the Special Supplemental Nutrition Program for Woman, Infants, and Children (WIC) ${ }^{3}$ despite provision of formula for partially and nonbreastfed infants. Coordinated WIC BF

Evaluation, Research, and Surveillance Unit, Division of Nutrition, New York State Department of Health, Albany, NY

Conflict of Interest Disclosure: The authors' conflict of interest disclosures can be found online with this article on www.jneb.org.

Address for correspondence: Lynn S. Edmunds, DrPH, MS, RD, Evaluation, Research, and Surveillance Unit, Division of Nutrition, New York State Department of Health, Rm 517, Riverview Center, 150 Broadway, Menands, NY 12204; Phone: (518) 402-7109; E-mail: Lynn.Edmunds@health.ny.gov

(C)2017 Society for Nutrition Education and Behavior. Published by Elsevier, Inc. All rights reserved.

promotion activities include BF counseling and education, support through the peer-counseling program, and the provision of the enhanced $\mathrm{BF}$ food package. The prevalence of BF among WIC-enrolled infants has been increasing for decades. However, disparities persist particularly regarding continuation beyond the early postpartum period. ${ }^{4,5}$ New York State (NYS) Pediatric Nutrition Surveillance System reports showed that among infants enrolled in the NYS WIC program in 2013, $81.2 \%$ initiated $\mathrm{BF}^{6}$ approaching the Healthy People 2020 objective of $81.9 \%$, and $52.0 \%$ breastfed for $\geq 3$ months. However, NYS Pediatric Nutrition Surveillance System reports also showed that $11.0 \%$ of all infants enrolled in 2013 were exclusively breastfed for 
$\geq 3$ months, $^{6}$ which was well below the Healthy People 2020 objective of $46.2 \%$. The substantial gaps between initiation/duration and exclusivity suggested that although mothers enrolled in NYS WIC want and attempt to breastfeed, they may face a unique set of challenges maintaining exclusive BF.

Infant feeding decisions are often complex and influenced by multiple interrelated factors. Potentially modifiable factors associated with exclusivity include psychosocial influences such as prenatal intention to breastfeed, ${ }^{7}$ social support and knowledge, attitudes, beliefs, and self-efficacy regarding BF., Moreover, some factors associated with exclusive $\mathrm{BF}$ are different from those associated with initiation and/or duration. ${ }^{10}$ These findings highlight the complex issues shaping infant feeding behavior and suggest that interventions to promote exclusive $\mathrm{BF}$ among WIC participants may need to target multiple salient barriers to exclusive BF.

Many promising interventions for BF promotion and support were composed of multiple components, spanned socioecological levels, and included a combination of prenatal and postnatal education, support, and/ or counseling ${ }^{11-13}$ offered during multiple visits by peer or professional health workers. ${ }^{11}$ Moreover, interventions that integrated components or processes into existing maternal care settings and systems (eg, routine clinic operations) may have been more beneficial and sustainable compared with interventions conducted by individual clinicians or researchers. ${ }^{13}$ These findings demonstrated that WIC clinic settings, with the capacity to provide lay and professional BF counseling throughout pregnancy and beyond, were uniquely positioned to intervene among populations with low exclusive BF rates.

In 2014, the NYS WIC program launched the You Can Do It (YCDI) initiative in 12 WIC clinics. This multicomponent intervention, which was originally developed by the Vermont WIC program, ${ }^{14}$ was designed to improve participants' knowledge, attitudes, confidence, and social support to breastfeed exclusively through a screening and tailored counseling protocol combined with peer counselor (PC) and professional support spanning the prenatal and early postpartum periods. ${ }^{14}$ Furthermore, YCDI built within, rather than beside, existing coordinated WIC BF promotion activities, while operationalizing and systematizing the personalized nutrition focus of the NYS WIC program. The objective of this article is to assess the impact of YCDI on exclusive BF prevalence across participating clinics while controlling for potential confounding factors and contextual effects.

\section{METHODS}

\section{Setting}

All 96 NYS WIC local agencies were given the opportunity to apply to participate. A total of 31 applications were received. All local agencies that applied were contacted and the lead manager was interviewed to gauge their ability to commit staff resources and make systems change to implement the intervention. Additional selection criteria included geographic location and BF performance. Twelve clinics were chosen to participate with the goal of ensuring geographic representation of 3 regions of the state as well as equal representation of clinics with low $(<10 \%)$, medium (10\% to $20 \%)$, and high ( $>20 \%$ ) rates of exclusive BF for $\geq 30$ days in 2013 . The study protocol was approved by the Institutional Review Board of the NYS Department of Health.

\section{Program Implementation}

Unique to NYS's implementation of YCDI was the formation of a yearlong learning community in which interdisciplinary teams of WIC staff from the 12 participating WIC clinics could learn from trainers and each other while setting agency-specific YCDI implementation goals. A total of 47 WIC staff members comprised 12 interdisciplinary teams, each included a BF coordinator, site manager, nutritionist(s), and BF PC(s). Teams participated in 8 learning community sessions, which were held from July, 2014 to January, 2015, where they were trained on how to implement the intervention, including training to improve BF counseling skills and competencies. Each team then conducted YCDI trainings with the remaining staff in their respective clinic. Participating clinics began enrolling prenatal women in September, 2014 and continued through January, 2015. To be eligible to enroll in the intervention, women had to have enrolled in WIC during the first trimester of pregnancy, be at least aged 18 years, speak English or Spanish, and indicate that they planned to breastfeed or be considering it. Eligible participants were provided an informed consent document and verbal consent was obtained from those who wanted to participate.

\section{Intervention Components}

A detailed description of the YCDI intervention implemented by the NYS WIC program was reported by Eldridge and colleagues. ${ }^{15}$ Only a brief explanation of relevant components is described here. A key intervention component was the use of the Breastfeeding Attrition Prediction Tool (BAPT), which is grounded in the Theory of Planned Behavior, ${ }^{16}$ to screen participating mothers and inform a tailored BF counseling plan targeting the following 3 domains: attitudes, social/professional support, and confidence. The BAPT was originally developed and validated for use during the postpartum period. ${ }^{17,18}$ A Spanish version of the tool, with some modification, was validated among low-income Mexican American women. ${ }^{19}$ The Vermont WIC program adopted a shortened version of the BAPT maintaining all 26 scored items ${ }^{19}$ and demonstrated that this 26-item BAPT was predictive of exclusive BF among WIC participants when administered during the first trimester. ${ }^{14}$ In this study, NYS WIC clinics used this 26item BAPT with consenting pregnant mothers during their initial first-trimester WIC visit. The BAPT is self-administered and takes approximately 10 minutes to complete. The complete scoring scheme of the BAPT is explained elsewhere. ${ }^{19}$ Based on an individuals' BAPT scores, a tailored counseling plan was developed to focus on domain(s) of concern (ie, negative attitudes, inadequate social/professional support, and/or low confidence) to be addressed during an individual followup session in the second trimester. For example, a woman scoring low in the attitudes domain would receive counseling from WIC nutrition educators specifically focused on building understanding regarding, for instance, the nutritional value of breast milk. 
Moreover, a total score of $\leq 20^{19}$ resulted in a coordinated care plan constructed through case conferencing among the WIC BF coordinator, PC(s), and nutrition educator(s). In the third trimester, participants attended a group discussion specifically designed to prepare mothers for delivery and the hospital stay. Within the first week after delivery, nutrition staff members and/or BF PCs contacted mothers twice to assess their BF experience and offer support and counseling. Attempts were made to certify both the infant and the mother in WIC within 24 weeks postpartum. Finally, collaborative efforts were made so that participants would continue to work with the same nutrition educator and PC throughout pregnancy and beyond to promote continuity of care.

\section{Evaluation Design}

This outcome evaluation was conducted using a quasi-experimental prospective cohort design (Figure 1). A baseline cohort was selected using the NYS WIC administrative data to identify a total of 844 pregnant women who enrolled in WIC during the first trimester at the same 12 clinics from September 1, 2013 through January 31, 2014 and demonstrated intention to breastfeed or remained open to the idea of $\mathrm{BF}$, as determined though routine screening questions asked by nutrition staff during WIC certification. Of these 844 participants, those who remained in WIC after birth comprised the baseline cohort with 688 mother-infant dyads. During the intervention enrollment period (ie, September 1, 2014 through January 31, 2015), a total of 826 pregnant women across the 12 WIC clinics met enrollment criteria and 418 pregnant women agreed to participate and completed a BAPT survey. Of those enrolled women, 362 mothers remained in WIC after birth and constituted the BAPT study cohort. Of the 408 pregnant women who declined to participate in the intervention, 347 mothers remained in WIC through birth and

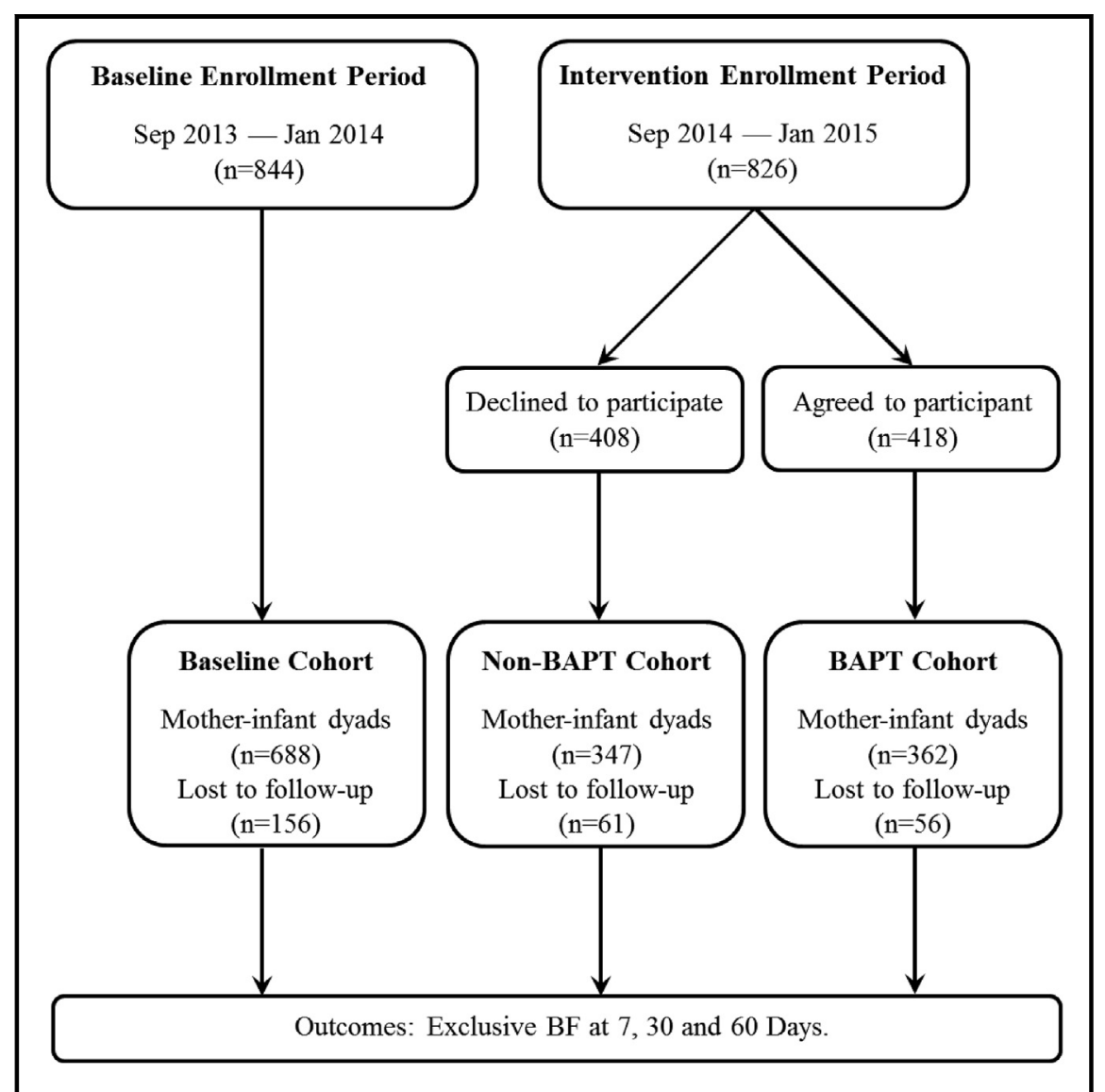

Figure 1. Diagram of the selection of 3 study cohorts at 12 Special Supplemental Nutrition Program for Women, Infants, and Children clinics in New York State. BAPT indicates Breastfeeding Attrition Prediction Tool; BF, breastfeeding. formed the non-BAPT study cohort. Although these women declined to participate in the intervention and thus were not assessed using the BAPT tool, they received the usual nutrition and BF counseling that each clinic routinely provided to its clients.

The primary outcome measure, duration of exclusive BF, was abstracted from WIC administrative data using the following question: How old was this child when he/she was first fed something other than breast milk? Responses were coded as dichotomous indicators of exclusive BF at 7, 30, and 60 days. These cutoffs for exclusivity measurement were chosen to be consistent with specific BF milestones within the NYS WIC program as well as important markers demonstrated in literature. ${ }^{20}$

Race/ethnicity was reported by the mother at WIC certification and categorized for analyses as non-Hispanic white (reported here as white), nonHispanic black (reported here as black), non-Hispanic other (reported here as other), or Hispanic. Because the other group is relatively small and diverse, the authors restricted analyses to white, black, and Hispanic mothers. Mother's education, also reported at the time of WIC certification, was categorized as less than high school, high school graduate or equivalent, or some college/college graduate. Income and household size were collected at the time of WIC certification and were categorized as the percentage of the federal poverty level. Parity was coded as first pregnancy vs previous pregnancies. The number of nutrition education contacts received during the prenatal period was abstracted from WIC administrative data and coded as $1-3$ or $\geq 4$ to distinguish between those who received the expected number of nutrition contacts (1-3) and those who received additional contacts.

\section{Statistical Analysis}

Differences in group characteristics were assessed using chi-square tests for independence for categorical variables or $t$ test for continuous variables. Outcomes were assessed using multiple logistic regressions, controlling for variables that differed significantly across cohorts. Evidence of effect modification indicated stratification by race/ethnicity. Prevalence estimates were compared across cohorts for each racial/ethnic group 
and within-group differences were assessed by Mantel-Haenszel chi-square tests for independence. Some clinics had few participants, which precluded the assessment of within-site and between-site differences.

To examine the impact of the intervention, differences in outcomes were assessed for the following cohorts (Figure 1): (1) BAPT (ie, intervention) vs baseline; (2) BAPT vs non-BAPT during the intervention period; and (3) non-BAPT vs baseline. In each instance, logistic regression analysis was used to assess the effect of the intervention, or group membership, on the outcome variables controlling for differences in maternal characteristics. Groups were further stratified by race/ethnicity and prevalence of each outcome was assessed across study cohorts. In the absence of a randomized control trial, within- and between-group comparisons were performed to test intervention effects and rule out alternative explanations. The first analysis (ie, BAPT vs baseline cohorts) provided a primary assessment of the effect of the intervention on BF outcomes. The second analysis (ie, BAPT vs non-BAPT cohorts) assessed whether exposure to the YCDI intervention was associated with outcomes above and beyond those observed in the same clinics during the same period. Finally, the third analysis (ie, non-BAPT vs baseline cohorts) assessed for temporal trends; evidence of no effect provided greater credibility for any intervention effect identified in the first analysis. All analyses were conducted using SAS software (version 9.4, SAS Institute, Inc, Cary, NC, 2002-2012).

\section{RESULTS}

Table 1 presents characteristics of the 3 study cohorts. There were statistically significant differences in mothers' ages across 3 cohorts: The non-BAPT mothers were approximately 1 year older than the women in the other 2 cohorts $(P=.005)$. The cohorts also differed in their race/ethnicity: The BAPT group had higher proportions of black and white women, but fewer Hispanic women, than either of the other 2 groups $(P<.001)$. Women in the BAPT cohort were less likely to be first-time mothers compared with the other 2 cohorts $(P=.02)$. Finally, a greater proportion of BAPT mothers had $\geq 4$ WIC nutrition
Table 1. Comparison of Mothers' Demographic Characteristics Among 3 Study Cohorts

\begin{tabular}{|c|c|c|c|c|}
\hline Characteristics & $\begin{array}{l}\text { Baseline } \\
(n=688)\end{array}$ & $\begin{array}{c}\text { Non-BAPT } \\
(n=408)\end{array}$ & $\begin{array}{c}\text { BAPT } \\
(n=362)\end{array}$ & $P^{\mathrm{a}}$ \\
\hline Mother's age, y (SD) & $28.6(5.6)$ & $29.7(6.0)$ & $28.9(5.8)$ & .005 \\
\hline $\begin{array}{l}\text { Race/ethnicity (\%) } \\
\text { Non-Hispanic white } \\
\text { Non-Hispanic black } \\
\text { Hispanic } \\
\text { Non-Hispanic other }\end{array}$ & $\begin{aligned} 76 & (11.1) \\
211 & (30.7) \\
352 & (51.2) \\
49 & (7.1)\end{aligned}$ & $\begin{array}{r}39(11.2) \\
75(21.6) \\
178(51.3) \\
55(15.6)\end{array}$ & $\begin{aligned} & 59(16.3) \\
& 122(33.7) \\
& 163(45.0) \\
& 18(5.0)\end{aligned}$ & $<.001$ \\
\hline $\begin{array}{l}\text { Education (\%) } \\
\text { Less than high school } \\
\text { High school } \\
\text { More than high school } \\
\text { Not specified }\end{array}$ & $\begin{array}{r}180(26.2) \\
274(39.8) \\
149(21.7) \\
85(12.4)\end{array}$ & $\begin{array}{r}96(27.7) \\
129(37.2) \\
69(19.9) \\
53(15.3)\end{array}$ & $\begin{array}{r}86(23.7) \\
160(44.2) \\
67(18.5) \\
49(13.5)\end{array}$ & .42 \\
\hline $\begin{array}{l}\text { Federal poverty level (\%) } \\
\text { 0 to } 100 \% \\
>100 \% \\
\text { Not specified }\end{array}$ & $\begin{array}{c}503(73.1) \\
154(22.4) \\
31(4.5)\end{array}$ & $\begin{array}{l}257(74.1) \\
76(21.9) \\
14(1.0)\end{array}$ & $\begin{array}{c}233(64.4) \\
100(27.6) \\
29(8.0)\end{array}$ & .01 \\
\hline $\begin{array}{l}\text { Parity (\%) } \\
\text { First pregnancy } \\
\text { Previous pregnancies }\end{array}$ & $\begin{array}{l}513(74.6) \\
175(25.4)\end{array}$ & $\begin{array}{r}278(80.1) \\
69(19.9)\end{array}$ & $\begin{array}{l}258(71.3) \\
104(28.7)\end{array}$ & .02 \\
\hline $\begin{array}{l}\text { Prenatal nutrition education (\%) } \\
1-3 \text { sessions } \\
\geq 4 \text { sessions }\end{array}$ & $\begin{array}{l}498(72.4) \\
190(27.6)\end{array}$ & $\begin{array}{r}256(73.8) \\
91(26.2)\end{array}$ & $\begin{array}{l}215(59.4) \\
147(40.6)\end{array}$ & $<.001$ \\
\hline
\end{tabular}

BAPT indicates Breastfeeding Attrition Prediction Tool.

${ }^{a}$ Chi-square test of independence used to assess significance.

Note: Twelve WIC clinics participated in the You Can Do it initiative launched by the NYS WIC program. The baseline cohort was composed of all eligible prenatal women certified at these 12 clinics between September 2013 and January 2014. The BAPT cohort was composed of all eligible and consenting prenatal women certified at these 12 clinics between September 2014 and January 2015. The non-BAPT cohort was composed of all eligible, but declined, prenatal women certified at these 12 clinics between September 2014 and January 2015. education contacts during the prenatal period than did their counterparts in the other 2 cohorts $(P<.001)$.

Multivariate logistic regression models adjusted for characteristics found significantly differences across groups (ie, race/ethnicity, age, parity, and number of prenatal WIC nutrition education sessions) in comparing: (1) BAPT vs baseline, (2) BAPT vs non-BAPT, and (3) non-BAPT vs baseline. Table 2 presents adjusted odds ratios (ORs) for each
Table 2. Effect of Study Cohort on EBF Outcomes at 7, 30, and 60 Days

\begin{tabular}{lccc} 
EBF & $\begin{array}{c}\text { BAPT }(\mathbf{n}=\mathbf{3 6 2}) \\
\text { vs Baseline } \\
\mathbf{( n = 6 8 8 )}\end{array}$ & $\begin{array}{c}\text { BAPT }(\mathbf{n}=\mathbf{3 6 2}) \\
\text { vs Non-BAPT } \\
(\mathbf{n}=\mathbf{4 0 8})\end{array}$ & $\begin{array}{c}\text { Non-BAPT }(\mathbf{n}=\mathbf{4 0 8}) \\
\text { vs Baseline } \\
\mathbf{( n = 6 8 8 )}\end{array}$ \\
\hline 7 & $1.6(1.2-2.3)^{\star}$ & $1.6(1.1-2.5)^{\star}$ & $0.98(0.7-1.4)$ \\
30 & $1.9(1.3-2.8)^{\star}$ & $1.6(1.0-2.5)^{\star}$ & $1.2(0.8-1.9)$ \\
60 & $1.8(1.2-2.7)^{\star}$ & $1.3(0.8-2.1)$ & $1.4(0.9-2.2)$ \\
\hline
\end{tabular}

BAPT indicates Breastfeeding Attrition Prediction Tool; EBF, exclusive breastfeeding.

Data are presented as adjusted odds ratios (95\% confidence intervals). Odds ratios and $P$ were generated using logistic regression, adjusted for race/ethnicity, mother's age, parity, and number of nutrition education sessions.

${ }^{\star} P<.05$. 


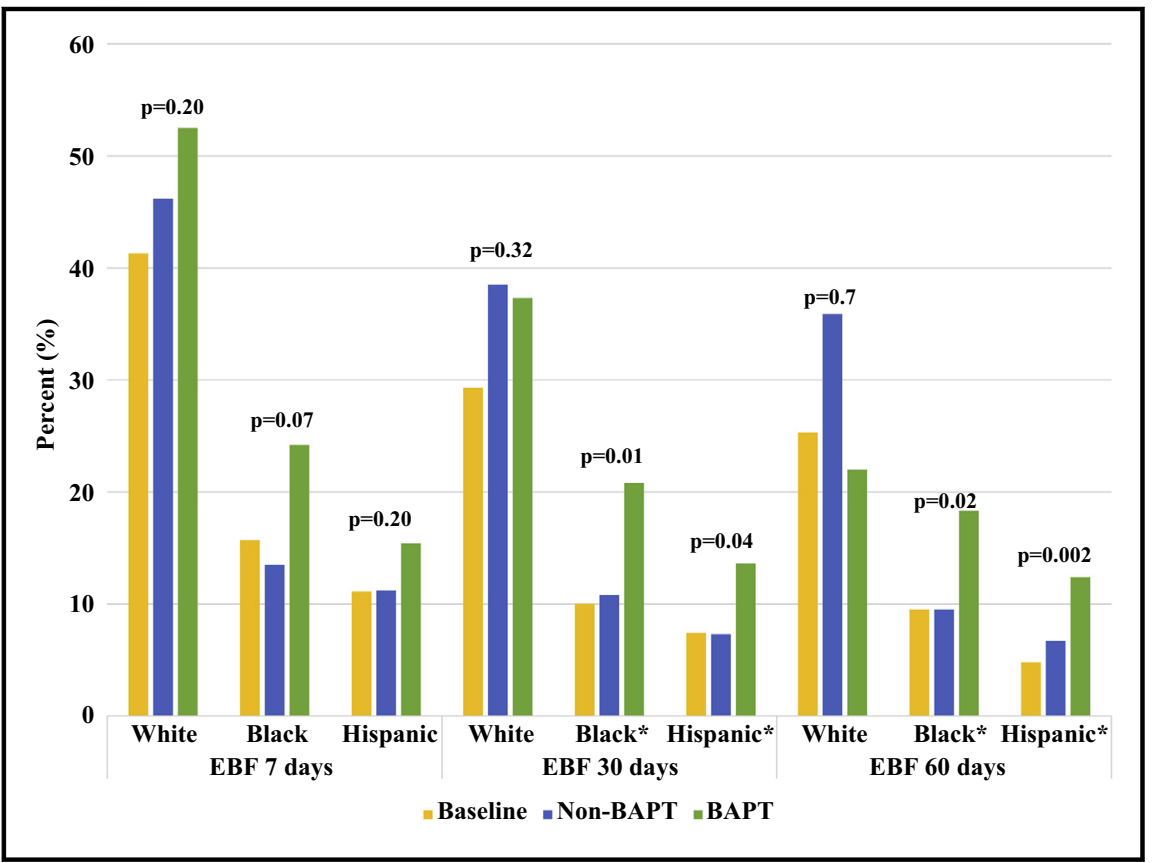

Figure 2. Comparison of exclusive breastfeeding (EBF) rates at 7,30 , and 60 days, by race/ethnicity and study cohort. White and black women are non-Hispanic; Hispanic women can be of any race. *Significant differences among study cohorts $(P<.05)$. BAPT indicates Breastfeeding Attrition Prediction Tool.

comparison by outcome. The BAPT participants were significantly more likely than were baseline participants to be exclusively BF at 7, 30, and 60 days (OR [95\% confidence interval], 1.6 [1.2-2.3], 1.9 [1.3-2.8], and 2.1 [1.4-3.1], respectively). Compared with the non-BAPT cohort, BAPT participants were significantly more likely to be exclusively BF at 7 and 30 days (OR [95\% confidence interval] 1.6 [1.1-2.5] and 1.6 [1.0-2.5], respectively), but not at 60 days. No significant differences were observed when comparing the non-BAPT and baseline groups.

Figure 2 presents the prevalence of exclusive BF at 7, 30, and 60 days stratified by race/ethnicity and study cohort. There were no statistical differences in the prevalence of exclusive $\mathrm{BF}$ at 7,30 , or 60 days among white women across the 3 study cohorts. Among black women, the prevalence of exclusive $\mathrm{BF}$ at 30 and 60 days was significantly higher among BAPT participants than for those in either the baseline cohort or the non-BAPT cohort. Similarly, Hispanic participants in the BAPT cohort had a significantly higher prevalence of exclusive $\mathrm{BF}$ at 30 and 60 days compared with their counterparts in both the baseline and nonBAPT cohorts.

\section{DISCUSSION}

The current study examined the impact of YCDI, a multicomponent intervention employing WIC resources and organizing WIC operations, on promoting exclusive BF in 12 NYS WIC clinics. Findings from this study suggested that YCDI participation was associated with improved exclusive BF within the first 2 months postpartum. These findings were consistent with those seen in the Vermont WIC program, in which higher exclusive $\mathrm{BF}$ rates were observed among mothers enrolled in the YCDI initiative in contrast to the respective comparison groups. ${ }^{14}$ Findings from this evaluation were also consistent with research, which suggests that interventions to promote BF with both prenatal and postnatal components are more effective at increasing exclusivity and duration than are interventions targeting women only during 1 period. ${ }^{12}$ Although a priority of the NYS WIC program is to promote and support $\mathrm{BF}$, this intervention may have been particularly effective in part because the YCDI intervention protocol increased the intensity of BF promotion: for example, by prioritizing $\mathrm{BF}$ counseling at each of 3 prenatal WIC visits and concentrating efforts on providing BF support to postpartum women within the first few days immediately after birth. Similarly, it is possible that striving for nutritionist and PC continuity across visits improved rapport between WIC staff and participants, thus supporting continuity of care ${ }^{15}$ and improving exclusive BF outcomes. ${ }^{21}$

Examination of BF outcomes by race and ethnicity revealed that the impact of the intervention was most profound among black and Hispanic women. It is possible that significant improvements in exclusive BF measurements among black and Hispanic women could be attributed, in part, to the assessment, evaluation, and tailored BF counseling sessions delivered, based on the BAPT scores. For example, the construct of self-efficacy, which is consistent with Social Cognitive Theory ${ }^{22}$ as well as the Theory of Planned Behavior, ${ }^{16}$ was assessed through the BAPT tool and addressed through tailored counseling in this intervention and has been shown to be positively associated with BF duration and exclusivity among African American women. ${ }^{23,24}$ Furthermore, support of a systematic assessment of factors known to affect BF behavior, accompanied by development of assessment-tailored BF assistance, is consistent with the National WIC Association's Six Steps to Achieve Breastfeeding Goals (step 6). ${ }^{3}$ In addition, studies indicated that provision of $\mathrm{BF}$ support within WIC settings may vary by race/ethnicity, and that black women were less likely to be offered lactation support. ${ }^{25,26}$ In this study, it was likely that adherence to the study protocol and perceived improvements in staff BF counseling competencies and confidence through learning community training, as reported by participating WIC staff at the end of the intervention, ${ }^{15}$ may have led to enhanced opportunities for all participants (in particular non-white women) to receive tailored BF support. On the other hand, the observation of little or no effect among white women, who had a relatively high prevalence of exclusive BF, suggested the potential of a ceiling effect, which can restrict and/or conceal the potential effectiveness of an intervention.

This study was subject to several limitations. First, the quasi-experimental, nonrandom assignment of participants to the BAPT and non-BAPT cohorts introduced the possibility of selection 
bias, which may threaten the validity of the findings. If the decision (ie, selection) to participate in the intervention itself was a positive determinant of the outcome measure, the effectiveness of the intervention would have been overestimated. Furthermore, if the BAPT cohort differed significantly from the non-BAPT cohort in ways that were not captured by the WIC administrative data, impact assessment of the intervention might have been misleading. Second, across all 3 study cohorts, $13.4 \%$ to $18.5 \%$ of eligible women (ie, enrolled in WIC during the first trimester within a certain time window, at least aged 18 years, speaking English or Spanish, and with intention to breastfeed) were excluded from this outcome analysis because they did not return to WIC after expected delivery for various reasons, which may have included fetal loss, improved socioeconomic status, or relocation, for example. Although statistical tests suggested that attrition rates differed somewhat among 3 study cohorts and by maternal age or race/ ethnicity, it was not feasible to examine the impact of loss to follow-up on any BF outcome measures. Furthermore, the disparate sizes of the clinic populations limited the ability to statistically control for clinic level variables which may have contributed to the findings. Additionally, it is not feasible to quantify the positive contribution or rank the importance of each component (eg, tailored counseling, group discussion, case conferencing, 1 week postpartum follow-up) to the overall success of the intervention.

\section{IMPLICATIONS FOR RESEARCH AND PRACTICE}

This evaluation study demonstrated positive outcomes of the YCDI initiative to promote exclusive $\mathrm{BF}$ duration among mothers enrolled in participating WIC clinics. You Can Do It was particularly effective in improving exclusive $\mathrm{BF}$ rates at 30 and 60 days among black and Hispanic mothers. The results of this outcome evaluation in the context of evaluating staff implementers' experiences ${ }^{15}$ suggest that this is an effective, adaptable, transferable, and likely sustainable initiative to improve BF exclusivity among women enrolled in the NYS WIC program. The YCDI initiative supports several of the Six Steps to Achieve Breastfeeding Goals ${ }^{3}$ set forth by the National WIC Association. Adoption and implementation of the initiative may support WIC clinic efforts to promote exclusive BF consistently as normative (step 1), provide hands-on training and procedures to support staff BF counseling confidence ${ }^{15}$ and competencies (step 5), and provide a straightforward protocol for assessing, evaluating, and then providing targeted assistance for BF-related participant concerns (step 6). However, limitations of this study highlighted the need for future research to determine effective contributions of specific intervention components given limited WIC staff time, clinic resources, and capacity to implement and sustain changes. In addition, participating clinics represented different regions of the state, diverse client environments, and with varying performance measures of BF promotion. Future work could assess how relevant aspects of the clinic setting influence both YCDI implementation and outcomes. An analysis linking clinic setting, implementation, and outcomes might improve understanding of why this intervention seems to be more effective among black and Hispanic women than white mothers.

\section{ACKNOWLEDGMENTS}

The authors of this article would like to thank the staff from the New York State WIC program, the participating clinics, and Cicatelli Associates, Inc, for their contribution to the planning and implementation of this intervention.

\section{REFERENCES}

1. Horta BL, Victora CG. Long-term Effects of Breastfeeding: A Systematic Review. Geneva, Switzerland: World Health Organization; 2013.

2. Victora CG, Bahl R, Barros AJD, et al. Breastfeeding in the 21st century: epidemiology, mechanisms, and lifelong effect. Lancet. 2016;387:475-490.

3. National WIC Association. Six Steps to Achieve Breastfeeding Goals for WIC Clinics. Washington, DC: National WIC Association; 2011.

4. Scanlon K, Rummer-Strawn L, Li R, Chen J, Molinari N, Perrine CG. Racial and ethnic differences in breastfeeding initiation and duration, by state- $\mathrm{Na}-$ tional Immunization Survey, United States, 2004-2008. MMWR. 2010;59: 327-334.

5. Allen JA, Li R, Scanlon K, et al. Progress in increasing breastfeeding and reducing racial/ethnic differences - United States, 2000-2008 births. MMWR. 2013;62: $77-80$.

6. Lee FF, Edmunds LS, Cong X, Sekhobo JP. Trends in breastfeeding among infants enrolled in the Special Supplemental Nutrition Program for Women, Infants and Children - New York, 2002-2015. MMWR. 2017;66: 610-614.

7. Tenfelde S, Finnegan L, Hill PD. Predictors of breastfeeding exclusivity in a WIC sample. J Obstet Gynecol Neonatal Nurs. 2011;40:179-189.

8. Chezem J, Friesen C, Boettcher J. Breastfeeding knowledge, breastfeeding confidence, and infant feeding plans: effects on actual feeding practices. J Obstet Gynecol Neonat Nurs. 2003;32: $40-47$.

9. de Jager E, Skouteris H, Broadbent J, Amir L, Mellor K. Psychosocial correlates of exclusive breastfeeding: a systematic review. Midwifery. 2013;29: 506-518.

10. Jones JR, Kogan MD, Singh GK, Dee DL, Grummer-Strawn LM. Factors associated with exclusive breastfeeding in the United States. Pediatrics. 2011;128:1117-1125.

11. Renfrew MJ, McCormick FM, Wade A, Quinn B, Dowswell T. Support for healthy breastfeeding mothers with healthy term babies. Cochrane Database Syst Rev. 2012;5:CD001141.

12. Rollins NC, Bhandari N, Hajeebhoy $\mathrm{N}$, et al. Why invest, and what it will take to improve breastfeeding practices? Lancet. 2016;387:491-504.

13. Chung M, Raman G, Trikalinos T, Lau J, Ip S. Interventions in primary care to promote breastfeeding: an evidence review for the U.S. Preventive Services Task Force. Ann Intern Med. 2008;149:565-582.

14. Bortree L, Decher L, Flynn K. Supporting Long Term Breastfeeding With the New WIC Food Packages-You Can Do It/WIC Can Help. Burlington, VT: Vermont Agency of Human Services; 2013.

15. Eldridge JD, Hartnett JO, Lee FF, Sekhobo JP, Edmunds LS. Implementing a WIC-based intervention to promote exclusive breastfeeding: challenges, 
S168 Edmunds et al

facilitators, and adaptive strategies. J Nutr Educ Behav. 2017;49(Suppl 2):S177-S185.

16. Montano D, Kasprzyk D. The Theory of Reasoned Action and the Theory of Planned Behavior. In: Glanz K, Rimer BK, Lewis M, eds. Health Behavior and Health Education. 3rd ed. San Francisco, CA: Jossey-Bass; 2002:67-98.

17. Janke J. Development of the BreastFeeding Attrition Prediction Tool. Nursing Res. 1994;43:100-104.

18. Dick M, Evans M, Arthurs J, et al. Predicting early breastfeeding attrition. J Hum Lact. 2002;18:21-28.

19. Gill S, Reifsnider E, Lucke J, Mann A. Predicting breast-feeding attrition: adapting the breast-feeding attrition prediction tool. J Perinat Neonat Nurs. 2007;21:216-224.

Journal of Nutrition Education and Behavior • Volume 49, Number 7S2, 2017

20. Wagner EA, Chantry CJ, Dewey KG, Nommsen-Rivers LA. Breastfeeding concerns at 3 and 7 days postpartum and feeding status at 2 months. Pediatrics. 2013;132:e865-e875.

21. Institute of Medicine Committee on Nutritional Status During Pregnancy and Lactation. Providing for the Continuity of Nutritional Care. Nutrition Services in Perinatal Care. 2nd ed. Washington, DC: National Academies Press; 1992.

22. Baranowski T, Perry CL, Parcel GS. How individuals, environments, and health behavior interact. In: Glanz K, Rimer BK, Lewis FM, eds. Health Behavior and Health Education, 3. San Francisco, CA: Jossey-Bass; 2002: 165-184.

23. McKinley E, Turner L. Increasing breastfeeding rates among African-
American women enrolled in the Special Supplemental Nutrition Program for Women, Infants, and Children: application of Social Cognitive Theory. Journal of Children and Poverty. 2017;23:95-118.

24. McCarter-Spaulding D, Gore R. Breastfeeding self-efficacy in women of African descent. J Obstet Gynecol Neonat Nurs. 2009;38:230-243.

25. Evans K, Labbok M, Abrahams SW. WIC and breastfeeding support services: does the mix of services offered vary with race and ethnicity? Breastfeeding Med. 2011;6:401-406.

26. Johnson A, Kirk R, Rosenblum K, Muzik M. Enhancing breastfeeding rates among African American women: a systematic review of current psychosocial interventions. Breastfeeding Med. 2015;10:45-62.

\section{Subscribe to JNEB Podcasts on iTunes to} automatically receive updates of newly posted materials on a variety of topics and listen to them while commuting or working at your desk.

https://itunes.apple.com/us/podcast/journal-nutrition-education/id437029195?ign-

$$
\mathrm{mpt}=\mathrm{uO} \% 3 \mathrm{D} 4
$$




\section{CONFLICT OF INTEREST}

The authors have not stated any conflicts of interest. 